\title{
Pareto-Optimization of HTS CICC for High-Current Applications in Self-Field
}

\author{
Giordano Tomassetti (D), Gianluca de Marzi, Giuseppe Celentano, Francesco Rizzo, \\ Andrea Augieri, and Antonio della Corte
}

\author{
ENEA C. R., 00044 Frascati, Italy \\ Correspondence should be addressed to Giordano Tomassetti; giordano.tomassetti@enea.it
}

Received 13 September 2017; Revised 23 November 2017; Accepted 7 December 2017; Published 28 January 2018

Academic Editor: Ying Xu

Copyright (c) 2018 Giordano Tomassetti et al. This is an open access article distributed under the Creative Commons Attribution License, which permits unrestricted use, distribution, and reproduction in any medium, provided the original work is properly cited.

\begin{abstract}
The ENEA superconductivity laboratory developed a novel design for Cable-in-Conduit Conductors (CICCs) comprised of stacks of 2nd-generation REBCO coated conductors. In its original version, the cable was made up of 150 HTS tapes distributed in five slots, twisted along an aluminum core. In this work, taking advantage of a 2D finite element model, able to estimate the cable's current distribution in the cross-section, a multiobjective optimization procedure was implemented. The aim of optimization was to simultaneously maximize both engineering current density and total current flowing inside the tapes when operating in self-field, by varying the cross-section layout. Since the optimization process involved both integer and real geometrical variables, the choice of an evolutionary search algorithm was strictly necessary. The use of an evolutionary algorithm in the frame of a multiple objective optimization made it an obliged choice to numerically approach the problem using a nonstandard fast-converging optimization algorithm. By means of this algorithm, the Pareto frontiers for the different configurations were calculated, providing a powerful tool for the designer to achieve the desired preliminary operating conditions in terms of engineering current density and/or total current, depending on the specific application field, that is, power transmission cable and bus bar systems.
\end{abstract}

\section{Introduction}

Today's commercial uses of the HTS technology are mainly limited to high-field magnets in the scientific field [1]. However, HTS cables are thought to have the potential to significantly reduce the amount of electricity lost in transmission and distribution in the electrical grid, estimated in about $10 \%$ of all generated electricity in the US, with this percentage growing year after year [2]. Also, in the perspective for commercial fusion reactor. HTS cables are considered the possible candidate magnetic system, because of the larger temperature margin in the coil section more exposed to nuclear heating that could be an advantage for the cryogenic and cooling system [1]. Moreover, the compactness of the HTS section would save more space for the blanket systems [3].

In 2013, ENEA proposed [4] a novel design of a HTS 2G wire-based CICC with twisted stacked tapes on slotted core
(Figure 1), allowing a fully industrial process implementation. The cable consists of 150 coated conductors, or 2G-wires, inserted in 5 stacks (30 tapes per stack) and included into ducts created in an aluminum core. The ducts have square cross-section to allocate $4 \mathrm{~mm}$ width tapes. The manufacturing process is fully compatible with available existing cabling technologies.

For such a configuration, the current density $J_{\text {eng }}$ takes into account the total cross-sectional area of the cable including superconducting and nonsuperconducting components, with the exclusion of the jacket section. Depending on the intended use of the HTS cable, the design should provide optimized value of the engineering current density $J_{\text {eng }}$ or of the total current $I_{C}{ }^{\text {cable }}$ flowing into the cable. For example, for high-field magnets a high $J_{\text {eng }}$ is a key feature, whereas for power transmission and/or distribution $J_{\text {eng }}$ does not constitute a real constraint. Other applications may require a compromise optimal trade-off between these two objectives. 


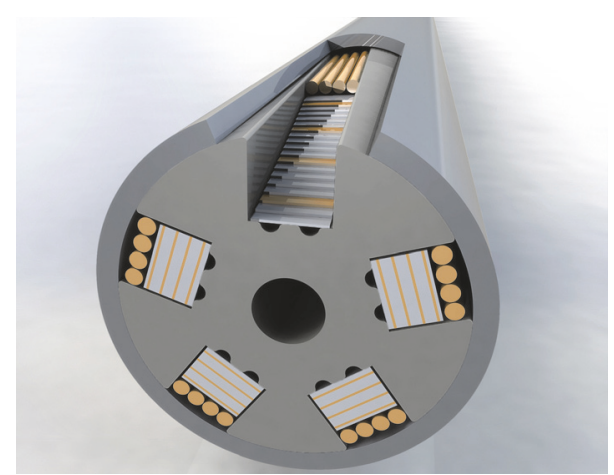

FIGURE 1: Artistic view of the assembled ENEA HTS 2G wire-based conductor.

Optimization is the mathematical process used to determine minima (or maxima) $\mathbf{x}^{*}$ of $l$ objective functions $F=$ $\left[F_{1}, \ldots, F_{l}\right]$ by varying a number of $n$ input design parameters $\mathbf{x}=\left[x_{1}, \ldots, x_{n}\right]$, so that a number $m$ of so-called constraint equations $g$ are simultaneously satisfied. In this sense, optimization becomes a crucial tool to achieve the best performance for the HTS cable as a function of the specific application. In particular, multiobjective optimization (also called Pareto-optimization) is useful in optimizing multiple, even conflicting, objectives giving full evidence to trade-off configurations using the Pareto frontier. The Pareto frontier is the graphical representation of the Pareto optimality conditions by showing the front of all the nondominated solutions. A solution is nondominated where another feasible solution better than the current one does not exist in some objective function without worsening other objective functions. On the contrary, dominated solutions are a set of design points performing worse than some other better points. Then, the Pareto frontier gives the designer an exact measure about how different choices in the design may produce changes in the trade-off among multiple conflicting objectives. So, depending on the specific application for the HTS cable, the designer may use the Pareto frontier to drive the design into a configuration which is best suited for one particular application rather than another. In other words, borrowing information from the Pareto frontier, the HTS cable design is customized depending on the relative "importance" of the engineering current density versus the total current.

In the frame of superconductors, Parametric optimization was recently used to design the highly homogeneous superconducting magnets for magnetic resonance imaging [5], or in beam-optics design of a gantry for carbon ions in cancer therapy accelerators [6], or in the optimal design of saturated iron-core superconducting fault current limiter [7], or to support designers with a guideline for selecting a suitable machine topology for a $10 \mathrm{MW}$ wind turbine application [8].

In this work, a novel approach to the design of the HTS cable cross-section, based on the Pareto optimality criterion, is proposed that may represent a useful tool for the designer to choose among different cable's configurations for electric power transmission, depending on the relative "importance" given to the current density $J_{\text {eng }}$ with respect to the total current $I_{C}{ }^{\text {cable }}$. The validity of this approach has been firstly assessed in self-field operating conditions by taking into account the effects of single tape $J_{c}$ dependence on magnetic field strength and direction at $77 \mathrm{~K}$.

In the following, Section 2.1 is dedicated to the description of the numerical model used to evaluate the current distribution within the cable's cross-section. In Section 2.2, the reasons for choosing the selected optimization algorithm are explained comparing pros and cons of the cutting edge optimization techniques, clarifying the advantage of the chosen approach. Section 2.3 is devoted to the detailed explanation of the optimization process in order to make a reproduction of this study possible. In Section 3.1, the results achieved with the optimization process are reported. In Section 3.2, these results are widely commented and discussed. And finally, Section 4 is a brief mention to possible future work based on this study even in different operating conditions.

\section{Materials and Methods}

2.1. Magnetostatic Model Description. Dealing with the complex problem of calculating the current distribution and the magnetic field inside the superconductor in a variety of geometries, such as those of stacked tapes configurations addressed in this paper, can be done using finite element method (FEM). Since the critical current $I_{c}$ of superconducting tapes depends on both the magnetic field's intensity and direction, it is of fundamental importance to optimize the tape distribution within the CICC in order to reduce the selffield degradation on $I_{c}$. Here, a $2 \mathrm{D}$ magnetostatic model of the commercial package COMSOL Multiphysics [9] was used based on the magnetic vector potential $A$, thus solving the following equation with the suitable boundary conditions:

$$
\nabla \times\left(\frac{1}{\mu} \nabla \times \mathbf{A}\right)=\mathbf{J}
$$

The self-field of stacked tapes has been investigated in a $2 \mathrm{D}$ cross-section of the cable under the assumption of ideal current sharing conditions (i.e., currents are free to redistribute with zero resistance in nonsuperconducting areas). In this approach, the stacks are assumed to be infinitely long and straight, and the current is perpendicular to the plane. Tapes have been modeled with their real dimensions: a thickness of $100 \mu \mathrm{m}$ and width of $4 \mathrm{~mm}$. For the description of the $J_{c}$ dependence on the local magnetic field $B$ and its direction $\theta$ (defined as the angle between the magnetic field and the normal vector to the superconducting tape), the following expression was used $[10,11]$ :

$$
\begin{aligned}
& I_{c}(B, \theta) \\
& =\frac{I_{c 0}}{1+\left(\sqrt{g^{2} \cos ^{2}\left(\theta-\theta_{0}\right)+\sin ^{2}\left(\theta-\theta_{0}\right)}\right)^{\alpha}\left(B / B_{0}\right)^{\beta}} .
\end{aligned}
$$

Although (2) is not based on a physical model, as the original anisotropic Kim model [10], it is able to reproduce the experimental data with greater accuracy. 


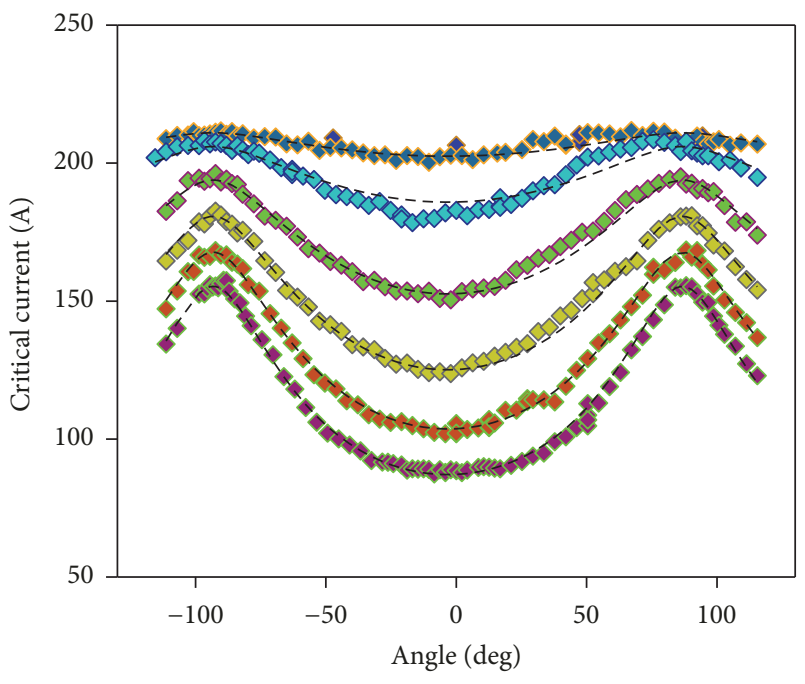

FIgURE 2: Dependence of the critical current on the angle of the applied magnetic field for several values of applied magnetic field. The symbols are experimental data; the lines show the results of the fits to (2).

$J_{c}$ as a function of angle $\theta$ at different magnetic field in liquid nitrogen was experimentally measured on patterned SuNAM SCN04150 tapes at $77 \mathrm{~K}$ (Figure 2). The experimental multiple data sets of Figure 2 were globally fit to (2) using the Igor Pro's Global Fit package with $I_{c 0}, g, \alpha$, and $\beta$ as global parameters and the field induction $B$ as a local parameter. Fitting parameters $I_{c 0}=214.56 \mathrm{~A}, B_{0}=499.1 \mathrm{mT}, g=3.895$, $\theta_{0}=-3.25^{\circ}, \alpha=0.99$, and $\beta=1.4$ were obtained.

Although being strongly dependent on the considered cross-section configuration, which varies along the optimization process, each single simulation took CPU time of about 300 sec on Pentium Dual Core e6300 @ 2.80 GHz RAM 4 GB.

In the original cable's cross-section configuration [4], the mesh is made up of 145,000 elements (see Figure 3) and the contour plot of the magnetic field intensity, in self-field conditions, is shown in Figure 4.

2.2. Optimization Algorithm Choice. Many methods have been proposed to solve optimization problems such as Mathematical Programming [12-14] and Nonlinear Programming [15-17]. Since the conversion of nonlinear problems into linear models is often not achievable, it is not always possible to apply Mathematical Programming algorithms. Whenever first- or second-order derivatives are available, Nonlinear Programming algorithms can be applied to solve the problem. Unfortunately, derivatives are often not easily available in practical problems. As a ground rule, Mathematical Programming and Nonlinear Programming methods constitute an effective gradient-based optimization set of algorithms for their intrinsic ability to find optimal solutions in few iterations. Nevertheless, gradient-based methods are not thought to deal with discontinuous objectives or design constraints or discrete design variables and they show a reduced exploration capability of the search domain with respect to evolutionary algorithms.

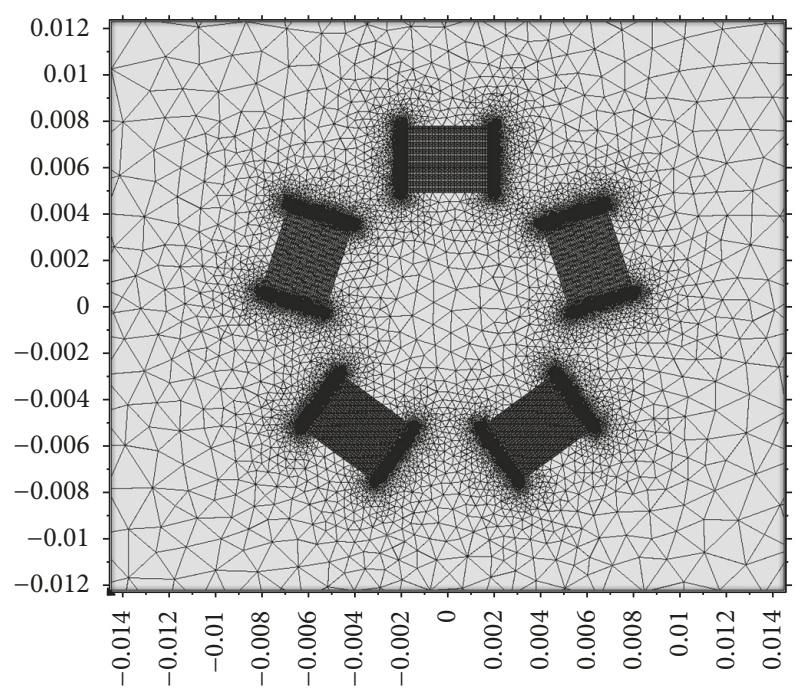

FIGURE 3: Finite element model mesh for the original configuration [4]: 30 tapes distributed in 5 slots with a spacing between consecutive tapes of $99 \mu \mathrm{m}$ and a radius of the inner tape of $4.95 \mathrm{~mm}$. The mesh consists of about 145,000 finite elements.

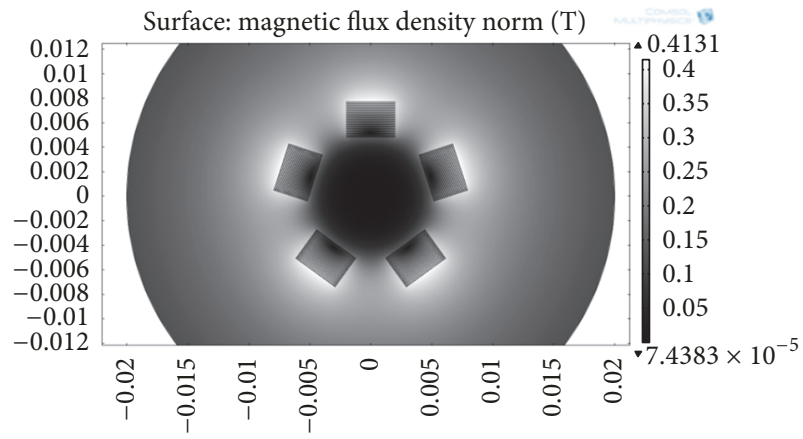

FIgURE 4: Contour plot in gray scale of the magnetic field intensity for the 30 tapes, 5 slots' original configuration [4] in self-field operating conditions.

The mentioned drawbacks of these methods have induced researchers to introduce another class of algorithms known as evolutionary algorithms (EAs). EAs imitate some natural mechanism of interaction among individuals with the aim of achieving a common goal. The common goal is something single agents could never be able to attain by themselves. So, cooperation plays a key role in detecting the optima. EAs such as Genetic Algorithms [18, 19] (GA) and the recent methods like Particle Swarm Optimization [20] (PSO) and Ant Colony Optimization [21] (ACO) are just some algorithms that have successfully exploited natural mechanisms for optimization purposes.

In this work, an enhanced version of the original PSO algorithm, called Minimized Computational Effort Particle Swarm Optimization (MCEPSO) [22], was used to the aim of maximizing current density at $77 \mathrm{~K}$ in self-field conditions with tapes arrangement in the cross-section as design parameters. MCEPSO has been structured in order to reduce, as much as possible, the computational effort of optimizations. 


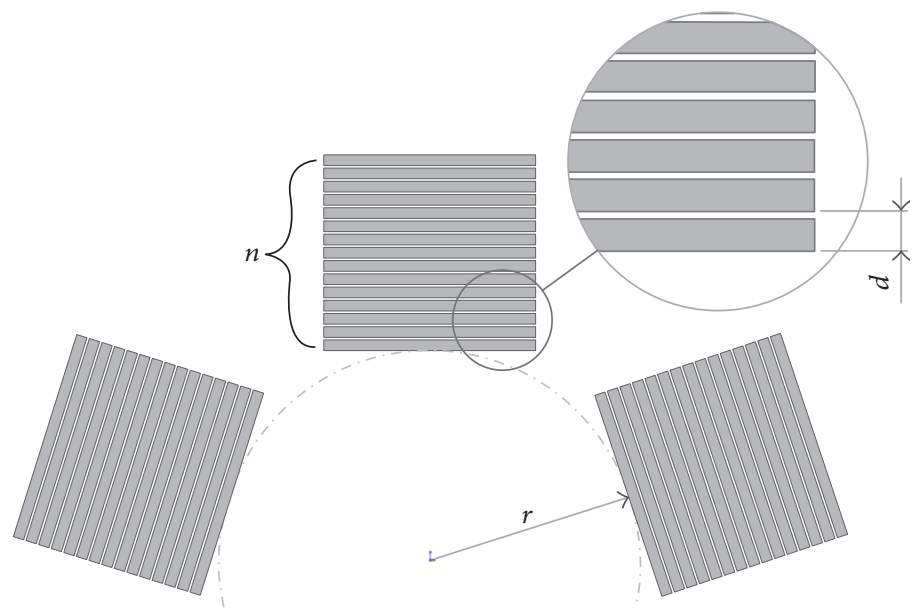

Figure 5: Simplified schematic of the cable's cross-section used to describe the design variables $n$ (number of tapes per slot), $d$ (spacing between two consecutive tapes), and $r$ (off-center distance of the inner tape) used in the optimization process.

This aspect is particularly useful when dealing with moderate to severe CPU simulation times. And also, MCEPSO introduces modification to the original PSO algorithm allowing dealing with constrained optimization and discrete design variables. As EAs typically involve thousands of function evaluations, it is substantially impossible to deal with computationally intensive simulations. MCEPSO proved to reduce significantly the number of required function evaluations to converge to an optimal design [22], thus allowing the optimization process in several applications where standard EAs may involve unaffordable computational times. MCEPSO was implemented to deal with single-objective optimization processes. However, as already mentioned, when dealing with cable's cross-section layout, two objectives are taken into consideration depending on the desired use of the cable: current density $J_{\text {eng }}$ and total flowing current $I_{C}{ }^{\text {cable }}$. In these cases MCEPSO is modified by implementing the so-called minimax approach [23], performing a certain number of optimizations while considering varying the relative "importance" (or "weight") of one objective with respect to the other.

With the mentioned simulation time of the order of magnitude of about $300 \mathrm{sec}$, the adoption of a proven fast convergent algorithm like MCEPSO was an obliged choice to keep the multiobjective optimization times for the 4 configurations $(3,4,5$, and 6 slots) within feasible values.

So, MCEPSO was selected with respect to gradient-based algorithms for its ability to deal with discrete variables, which are an intrinsic aspect of the considered optimization problem. MCEPSO was preferred to other EAs because it demonstrated, on widely approved benchmark test problems [22], being able to find the optimal configuration in a minimized number of FE simulations, which may become a bottleneck when dealing with simulation times of the order of about $6 / 7$ minutes in the frame of a multiobjective search (typically involving hundreds of thousands of FE function evaluations).

2.3. Optimization Process Description. The optimization variables considered in this work were the number of tapes $n$
TABLE 1: Design constraints for the off-center distance.

\begin{tabular}{lcc}
\hline Number of slots & Lower limit $(\mathrm{mm})$ & Upper limit $(\mathrm{mm})$ \\
\hline 3 & 4.8 & 9.6 \\
4 & 7.7 & 15.4 \\
5 & 10.4 & 20.8 \\
6 & 12.9 & 25.8 \\
\hline
\end{tabular}

Side constraints posed for design variable off-center distance $r$.

per slot, the off-center distance $r$ of the innermost tape, and intertape spacing $d$ between two consecutive tapes, as graphically described in Figure 5. The aim of the optimization process is to maximize, at the same time, both the current density $J_{\text {eng }}(d, n, r)$ and the total current $I_{C}{ }^{\text {cable }}(d, n, r)$ which are both dependent on the mentioned design variables $d, n$, $r$, keeping the number of slots $n_{\text {slots }}$ as a parameter.

The simultaneous effect of varying the entire set of the design variables (the off-center distance $r$, the intertape spacing $d$, and the number of tapes per slot $n$ ) and the number of slots $n_{\text {slots }}$ on the total current $I_{C}{ }^{\text {cable }}$ and current density $J_{\text {eng }}$ is not trivial to be estimated in advance and needs an optimization tool to be exhaustively explored.

The multiobjective optimization was repeated, varying the number of slots $n_{\text {slots }}$ in the cable (from 3 to 6 slots) and with the tapes' widths kept fixed at $4 \mathrm{~mm}$ and considering SuNAM tapes. SuNAM tapes were characterized as described in [11] in terms of the dependence of the critical current when varying the angle of the applied magnetic field. Among the design variables, the off-center distance $r$ was considered to be continuous whereas the number of tapes in the stack $n$ and intertape spacing between consecutive tapes $d$ were discrete variables. For the sake of precision, the number of tapes $n$ could vary between 22 and 80 while the intertape spacing $d$ could assume the following values: 149, 170, 190, 210, 230, 250, 270, or $300 \mu \mathrm{m}$. Off-center distance of the inner tape $r$ was constrained to vary continuously between a lower and an upper value defined as a consequence of manufacturing constraints for the cable's core, as detailed in Table 1. Based on 
previous design experiences and industrial feasibility limits [4], the side limits for the other design variables were decided, as detailed previously and in Table 1 .

The multiobjective optimization, as detailed in the previous part of this section, is mathematically summarized by the following equation:

$$
\begin{array}{ll}
\max & {\left[I_{c}^{\text {cable }}(d, n, r), J_{\text {eng }}(d, n, r)\right]} \\
\text { with } & d=[149 \mu \mathrm{m}, \ldots, 300 \mu \mathrm{m}] \\
& r_{\min }\left(n_{\text {grooves }}\right) \leq r \leq r_{\max }\left(n_{\text {grooves }}\right) \\
& n=[22 \div 80]
\end{array}
$$

\section{Results and Discussion}

3.1. Results. As explained, the optimization was repeated varying the cable's configuration $\left(n_{\text {slots }}\right)$ from 3 to 6 slots in order to compare the results obtained in terms of $J_{\text {eng }}$ and $I_{C}$ cable. Results are summarized in the Pareto frontiers depicted in Figure 6. The obtained Pareto frontiers allow the designer to evaluate the incremental benefits associated with the enhancement of the design optimality. In other terms, the Pareto frontiers play a fundamental role in the design since the designer can evaluate exactly how much more cost is incurred on a certain objective if another objective was favored by a certain amount. It is important to underline that any of the infinite solutions lying on the Pareto frontiers is an optimal solution for the HTS cable design. Moving from one side to the other on a single Pareto frontier, the relative "importance" of one objective with respect to the other varies from $0 \%$ to $100 \%$. This means that optimal solutions on the left hand of the single Pareto line are achieved giving $100 \%$ of relative "weight" to total current $\sum I$ (and $0 \%$ to the current density $J_{\text {eng }}$ ). On the contrary, optimal points on the right hand are characterized by $100 \%$ of relative importance given to the current density $J_{\text {eng }}$ (and $0 \%$ to the total current $\left.I_{C}{ }^{\text {cable }}\right)$. Any intermediate point on each Pareto frontier is yet optimal but with a different relative percentage of "importance" between the two objectives. Each point belonging to the Pareto frontiers is also characterized by the respective optimal values of the design variables $(n, r$, and $d$ ) that are not represented in the Pareto plot which is a representation in the objective domain ( $J_{\text {eng }}$ versus $I_{C}{ }^{\text {cable }}$ ) rather than in the design variables domain.

The optimal solutions have also been represented, in Figures 7-10, as a function of the relative "weight" given along the multiobjective optimization process (upper panels) and in terms of the design variables versus the same "weight" (lower panels).

Moving from the left to the right in the design variables' plots (from Figures 7-10, lower plots) corresponds to moving from the right bottom to the left upper side of the objective's domain represented in Figure 6. As Figure 6 only represents the infinite set of optimal solutions in the objective's domain,

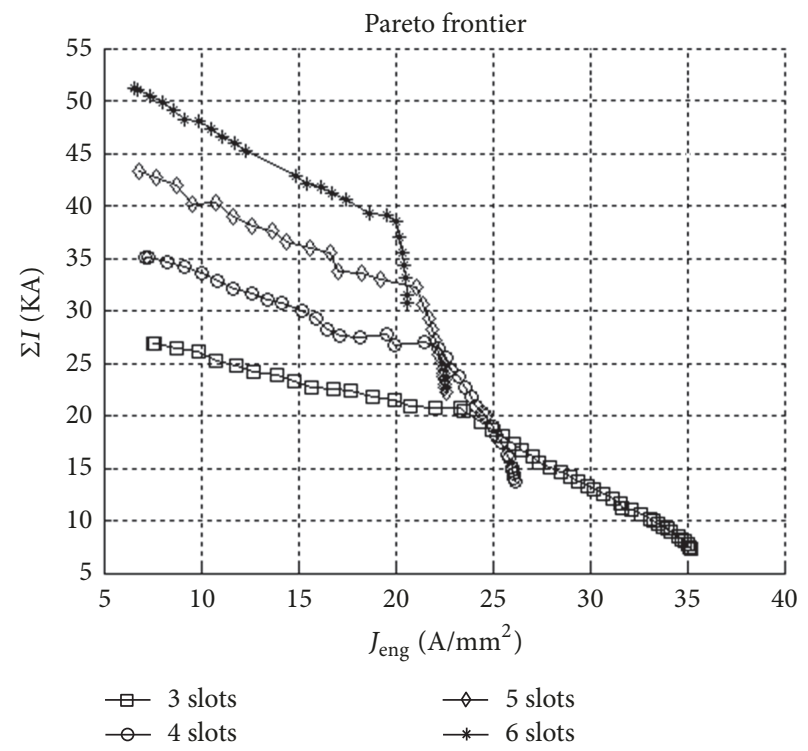

Figure 6: Pareto frontier, in the objectives domain, representing the full set of optimal solutions found for 3, 4, 5, and 6 slots' configurations.

Figures $7-10$ are useful to interpret these infinite optimal sets in terms of the respective the design variables.

3.2. Discussion. Under the simplifying assumptions introduced in the FEM model, by means of the multiobjective optimization process clear trends were identified to assist the designer's choice in the definition of the best cross-section configuration for HTS CICC cables.

In general terms, from Figure 6, it seems that optimized values for $I_{C}{ }^{\text {cable }}$ are obtainable by increasing the number of slots $n_{\text {slots }}$ up to 6 (upper left side of the plot). On the contrary, highest values for the $J_{\text {eng }}$ are achieved using the 3slot configurations (lower right side of the plot).

Also, in the central area of the plot in Figure 6, the same optimal configuration, in terms of the objectives $\left(I_{C}{ }^{\text {cable }}\right.$ and $\left.J_{\text {eng }}\right)$ is achievable with different cross-section configurations, as the Pareto fronts intersect each other. In these cases, the cost of the different layouts may represent the decisive criteria for the layout's choice.

Taking into consideration plots from Figures $7-10$, it is firstly clear that a more closely spaced configuration allows an increase in the cable's current density $J_{\text {eng }}$ while a wider tape distribution in space is more favorable to larger current values. In fact, the cable's cross-sections layouts which are closer to the origin in plots from Figures 7-10 are characterized by higher values of the current density $J_{\text {eng }}$ and lower values of the total current $I_{C}{ }^{\text {cable }}$, while also being characterized by smaller values of all the three design variables $n, r$, and $d$. In the same plots, moving towards the right hand corresponds to increasing the relative "importance" of $I_{C}{ }^{\text {cable }}$ with respect to $J_{\text {eng }}$ in the multiobjective optimization. As a consequence, moving towards the right hand of the plots causes the optimal solutions to be characterized by increased value of $I_{C}{ }^{\text {cable }}$ and 


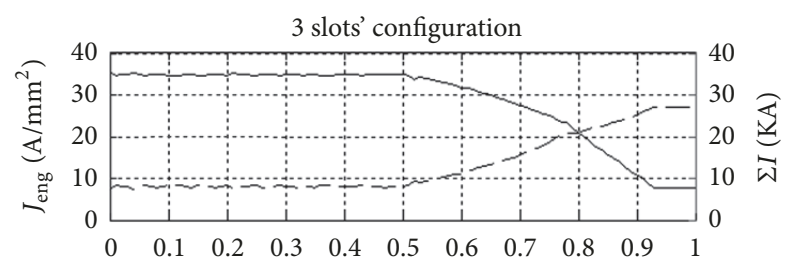

$-J_{\text {eng }}$
$--\Sigma I$

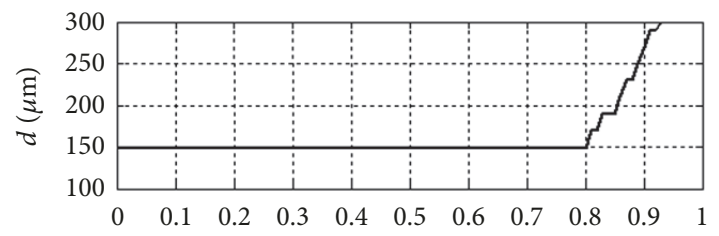

_ Intertape spacing " $d$ "

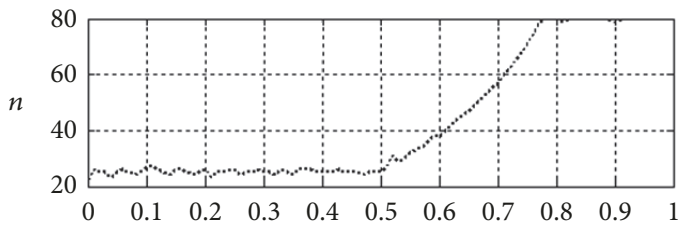

......... Numb. of tapes per slot “ $n$ "

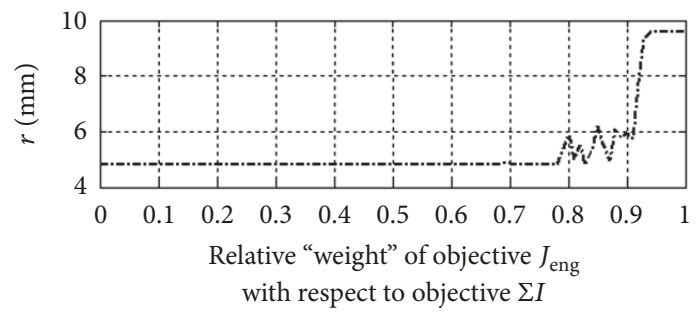

-.-- Inner tape off-center dist. " $r$ "

Figure 7: Pareto frontier represented as a function of the relative "weight" of one objective with respect to the other for the 3 slots' configuration $\left(n_{\text {slots }}=3\right)$. Top panel: values of the two objectives. Bottom panels: values of the respective design variables.

smaller values of $J_{\text {eng, }}$, also corresponding to design variables $n, r$, and $d$ to increase to their respective upper limits.

Secondly, all the plots from Figures 7-10 are characterized by two plateaus: the first one on the left hand of the plots, where design variables have the lowest values, and the second on the right hand, for the highest values of $n, r$, and $d$. Along these two plateaus, the values of the design variables are kept fixed by the optimizer so the corresponding objective values on Figure 6 are coincident multiple points laying exactly on the right and left end of the Pareto lines. This means that, despite the fact that the optimizer is increasing the relative "importance" of $I_{C}$ cable with respect to $J_{\text {eng }}$, the best configuration is still the same as it was considering when giving no "importance" at all to $I_{C}$ cable with respect to $J_{\text {eng. }}$. As the relative "importance" is further increased, the very first design variable to be changed by the optimizer is the number
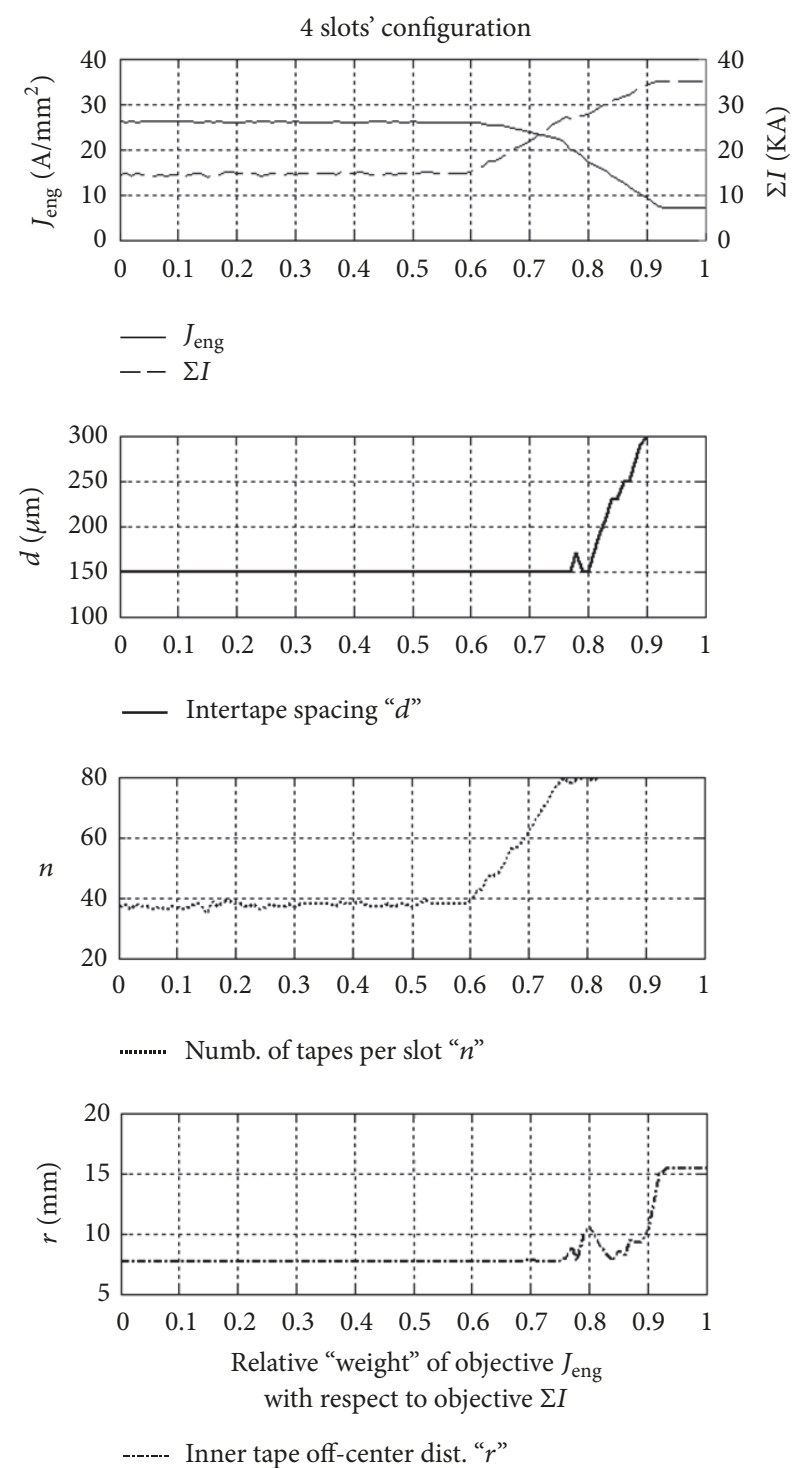

FIGURE 8: Pareto frontier represented as a function of the relative "weight" of one objective with respect to the other for the 4 slots' configuration $\left(n_{\text {slots }}=4\right)$. Top panel: values of the two objectives. Bottom panels: values of the respective design variables.

of tapes $n$. When the relative importance is further increased, also the remaining two design variables $r$ and $d$ are increased.

The presence of the two mentioned plateaus in plots from Figures 7-10 also allows understanding that the full range of variability for the objectives, represented in Figure 6 by all the intermediate points between the two side ends of each Pareto line, corresponds to the intermediate values in the lower plots of Figures 7-10, where the design variables are increased from each lower plateau to each higher one. This means that an effective change in the objectives' balance is achieved giving a minimum threshold of relative "importance" of to $I_{C}{ }^{\text {cable }}$ with respect to $J_{\text {eng }}$. And this threshold seems to be changing when considering the 3 slots' configuration (where the threshold is about 0.5 ), the 4 slots' configuration (threshold is about 0.65 ), 


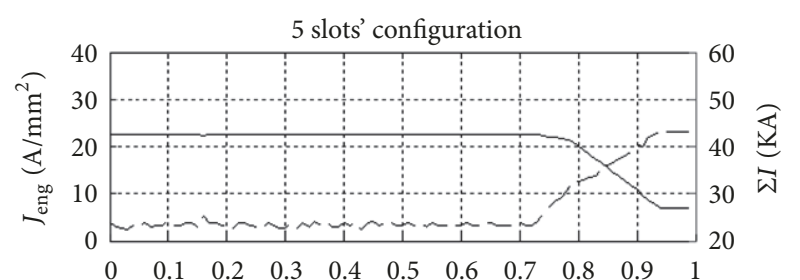

$$
\begin{aligned}
& -J_{\text {eng }} \\
& --\Sigma I
\end{aligned}
$$

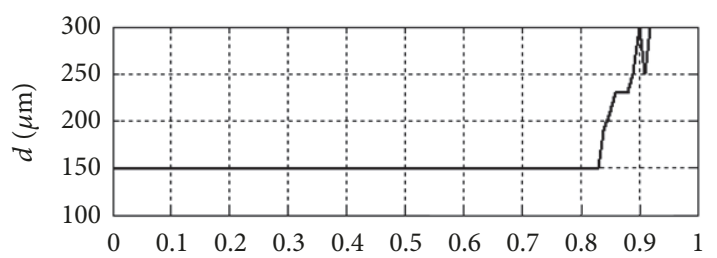

_ Intertape spacing “ $d$ ”

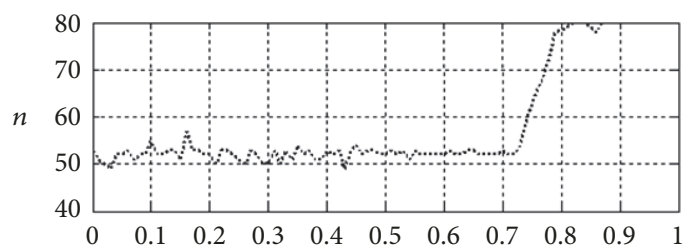

......... Numb. of tapes per slot " $n$ "

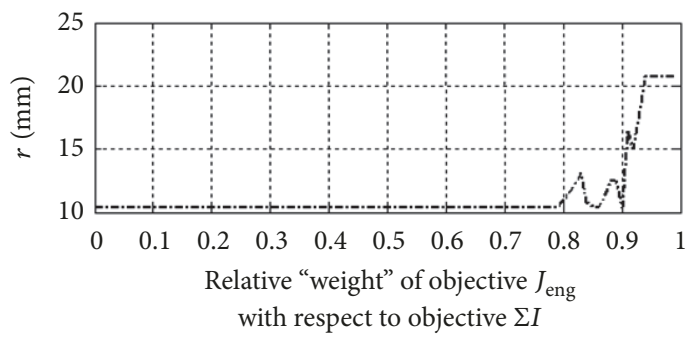

----- Inner tape off-center dist. " $r$ "

Figure 9: Pareto frontier represented as a function of the relative "weight" of one objective with respect to the other for the 5 slots' configuration $\left(n_{\text {slots }}=5\right)$. Top panel: values of the two objectives. Bottom panels: values of the respective design variables.

the 5 slots' configuration (threshold is about 0.75 ), and the 6 slots' configuration (threshold is about 0.65 ).

Also it is interesting to notice that, as the number of slots $n_{\text {slots }}$ increases in Figures 7-10, the left plateau for the number of tapes (design variable $n$ ) increases from about 25 (Figure 7) to about 58 in Figure 10. On the contrary, the left plateau for the design variable $d$ is fixed to about $150 \mu \mathrm{m}$, regardless of the number of slots $n_{\text {slots }}$.

A change of slope is easily detectable in Figure 6 for each of the Pareto contours. Focusing the attention on the 6 slots' Pareto line in Figure 6, the change of slop is detected for $I_{C}$ cable $\approx 38 \mathrm{KA}$ and $J_{\text {eng }} \approx 20 \mathrm{~A} / \mathrm{mm}^{2}$. The corresponding point in the upper panel of Figure 10 is characterized by an abscissa (relative "importance" of to $I_{C}{ }^{\text {cable }}$ with respect

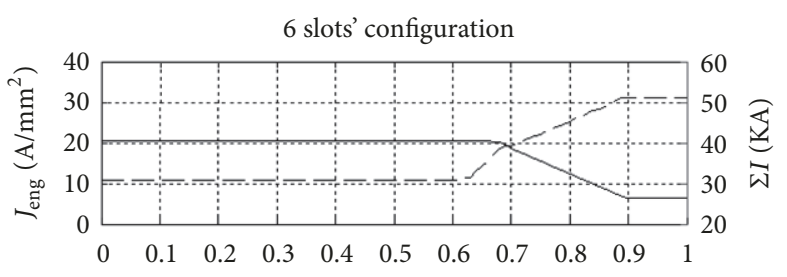

$$
-J_{\text {eng }}
$$

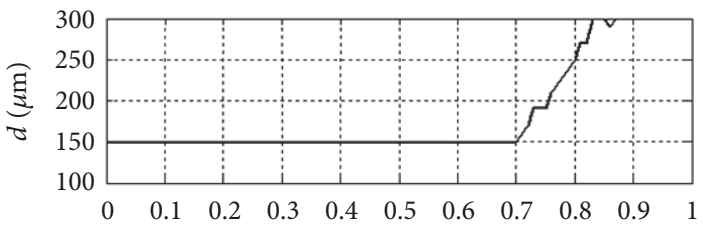

— Intertape spacing " $d$ "

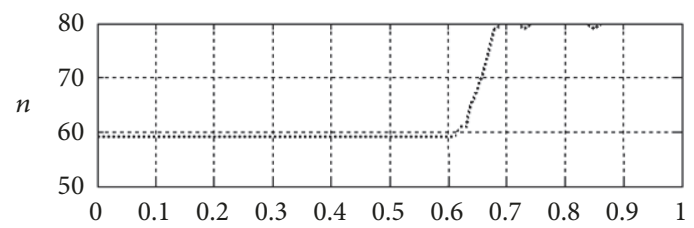

........... Numb. of tapes per slot " $n$ "

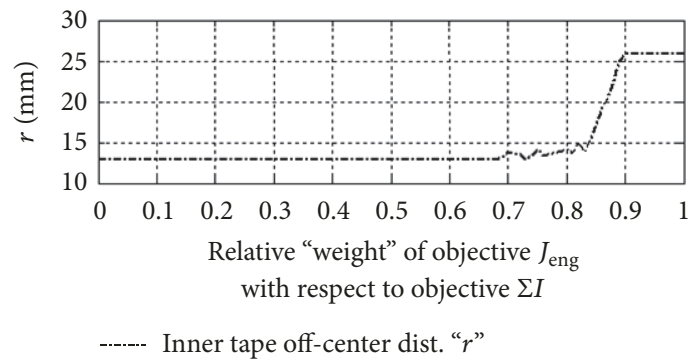

FIGURE 10: Pareto frontier represented as a function of the relative "weight" of one objective with respect to the other for the 6 slots' configuration $\left(n_{\text {slots }}=6\right)$. Top panel: values of the two objectives. Bottom panels: values of the respective design variables.

to $J_{\text {eng }}$ ) of about 0.7 . For this specific value of the abscissa, the third panel from the top in Figure 10 indicates that design variable $n$ has reached the top plateau and cannot be increased anymore by the optimizer. From now on, to further increase the total current $I_{C}{ }^{\text {cable }}$ (at the expense of $J_{\text {eng }}$ ) in Figure 6, the optimizer will increase the other two design variables: in particular, for an abscissa of 0.7 , the optimizer starts to increase design variable $d$ (second panel from the top in Figure 10) and then, for an abscissa of about 0.83 , it starts increasing also $r$. So, in Figure 6, as long as the optimizer has the possibility of increasing the number of tapes $n$, an increase in total current $I_{C}{ }^{\text {cable }}$ is achieved with a steep slope with respect to $J_{\text {eng. }}$. When the number of tapes $n$ reaches its top side range value, the total current $I_{C}{ }^{\text {cable }}$ continues to increase but with a much smaller slope than before. This residual increase of $I_{C}$ cable , from the Pareto knee to the max value of 
$I_{C}{ }^{\text {cable }}$ (about $52 \mathrm{KA}$, for the 6 slots' configuration in Figure 6), is due to the reduction of the self-field. Similar conclusions may be drawn for the 3,4 , and 5 slots' configurations in Figure 6 and the corresponding Figures 7, 8, and 9, although the change of slope is less emphasized as the number of slots is decreased, because the total number of tapes gets reduced as well.

\section{Conclusions}

In this work, the multiobjective optimization of the ENEA HTS cable's cross-section in self-field operating conditions was studied, drawing novel and nontrivial conclusions about the influence of design parameters on the cable's electrical features. In particular, a parametric 2D FEM model of the cable was implemented to predict $J_{\text {eng }}$ and $I_{C}{ }^{\text {cable }}$, also considering the experimental angular dependence of $I_{C}{ }^{\text {cable }}$ from both the magnetic field's intensity and direction. Using a parametric model allowed FEM/optimizer coupling. Considering the bottleneck represented by the huge number of FEM analyses needed to perform a multiobjective optimization, the optimizer was selected for its effectiveness and for being able to deal with multiple objectives and discrete design variables.

The proposed design methodology may be applied to supply useful instructions to the designer about the influence of the cable's layout on the respective electrical characteristics, in order to attain improved performance, in self-field conditions.

In particular, in those applications where available space represents a constraint and consequently high $J_{\text {eng }}$ are required, a more closely spaced configuration is to be preferred by the designer. In all other cases, such as typically in the case of transport cables, the number of slots can be increased, which results in a wider space distribution of superconducting tapes.

Future work will consist in modifying the FEM model in order to consider different operating conditions, like infield working conditions, to draw conclusions for the designer about the optimal cable's cross-section for high-field magnets.

\section{Conflicts of Interest}

The authors declare that there are no conflicts of interest regarding the publication of this paper.

\section{Acknowledgments}

The authors would like to thank A. Anemona for HTS CICC drawings and S. Chiarelli for help in designing constraints definitions.

\section{References}

[1] C. Senatore, M. Alessandrini, A. Lucarelli, R. Tediosi, D. Uglietti, and Y. Iwasa, "Progresses and challenges in the development of high-field solenoidal magnets based on RE123 coated conductors," Superconductor Science and Technology, vol. 27, no. 10, Article ID 103001, 2014.
[2] A National Effort to Introduce New Technology into the Power Delivery Infrastructure, Superconductivity Program for Electric Systems, Office of Electricity Delivery and Energy Reliability, U.S. Department of Energy, http://energy.gov/sites/prod/files/ oeprod/DocumentsandMedia/cable_overview2.pdf.

[3] T. Brown, L. Bromberg, and M. Cole, "Results of Compact Stellarator engineering trade studies," in Proceedings of the 2009 23rd IEEE/NPSS Symposium on Fusion Engineering - SOFE, pp. 1-4, San Diego, CA, USA, June 2009.

[4] G. De Marzi, N. C. Allen, L. Chiesa et al., "Bending Tests of HTS Cable-In-Conduit Conductors for High-Field Magnet Applications," IEEE Transactions on Applied Superconductivity, vol. 26, no. 4, 2016.

[5] Z. Ni, Q. Wang, F. Liu, and L. Yan, "A homogeneous superconducting magnet design using a hybrid optimization algorithm," Measurement Science and Technology, vol. 24, Article ID 125402, 2013.

[6] J. Kim and M. Yoon, "Design study of a superconducting gantry for carbon beam therapy," Journal of the Korean Physical Society, vol. 69, p. 1048, 2016.

[7] A. Dey and A. B. Choudhury, "A comparative study between scalarization approach and Pareto approach for multi-objective optimization problem using Genetic Algorithm (MOGA) formulated based on superconducting fault current limiter," in Proceedings of the 1st IEEE International Conference on Power Electronics, Intelligent Control and Energy Systems, ICPEICES 2016, India, July 2016.

[8] D. Liu, H. Polinder, A. B. Abrahamsen et al., "Optimization and comparison of superconducting generator topologies for a 10 MW wind turbine application," International Journal of Applied Electromagnetics and Mechanics, vol. 53, pp. S191-S202, 2017.

[9] Comsol Multiphysics, 2014, http://www.comsol.com.

[10] P. M. Leys, M. Klaeser, F. Schleissinger, and T. Schneider, "Analysis of the anisotropic critical current behaviour of HTS coated conductors," Journal of Physics: Conference Series, vol. 507, no. 2, Article ID 022013, 2014.

[11] E. Pardo, M. Vojenčiak, F. Gömöry, and J. Šouc, "Low-magneticfield dependence and anisotropy of the critical current density in coated conductors," Superconductor Science and Technology, vol. 24, no. 6, Article ID 065007, 2011.

[12] A. Ben-Tal and A. Nemirovski, "Robust optimization - Methodology and applications," Mathematical Programming, vol. 92, no. 3, pp. 453-480, 2002.

[13] D. Bertsimas, D. Pachamanova, and M. Sim, "Robust linear optimization under general norms," Operations Research Letters, vol. 32, no. 6, pp. 510-516, 2003.

[14] Y. Kanno e and I. Takewaki, "Evaluation and maximation of robustness of trusses by using semidefinite programming," in Proceedings of the 6th World Congress on Structural and Multidisciplinary Optimization (WCSMO), Rio de Janeiro.

[15] X. Du and W. Chen, "Towards a better understanding of modeling feasibility robustness in engineering design," Journal of Mechanical Design, vol. 122, no. 4, pp. 385-394, 2000.

[16] D. Indraneel, "Robustness optimization for constrained nonlinear programming problems," Engineering Optimization, vol. 32, no. 5, pp. 585-618, 2000.

[17] I. Doltsinis and Z. Kang, "Robust design of structures using optimization methods," Computer Methods Applied Mechanics and Engineering, vol. 193, no. 23-26, pp. 2221-2237, 2004.

[18] J. H. Holland, Adaptation in Natural and Artificial Systems: An Introductory Analysis with Applications to Biology, Control, and 
Artificial Intelligence, University of Michigan Press, Oxford, UK, 1975.

[19] D. E. Goldberg, "Genetic algorithms in search, optimization, and machine learning," Choice Reviews Online, vol. 27, no. 02, pp. 27-0936-27-0936, 1989.

[20] Y. Shi and R. C. Eberhart, "A Modified Particle Swarm Optimizer," in Proceedings of the International Conference on Evolutionary Computation, pp. 69-73, IEEE Service Center, Anchorage, AK, USA, 1998.

[21] M. Dorigo, Dorigo Optimization, Learning and Natural Algorithms [Ph.D. thesis], Politecnico di Milano, Italy, 1992.

[22] G. Tomassetti and L. Cagnina, "Particle swarm algorithms to solve engineering problems: a comparison of performance," Journal of Engineering, vol. 2013, Article ID 435104, 13 pages, 2013.

[23] H.-D. Joos, "A methodology for multi-objective design assessment and flight control synthesis tuning," Aerospace Science and Technology, vol. 3, no. 3, pp. 161-176, 1999. 

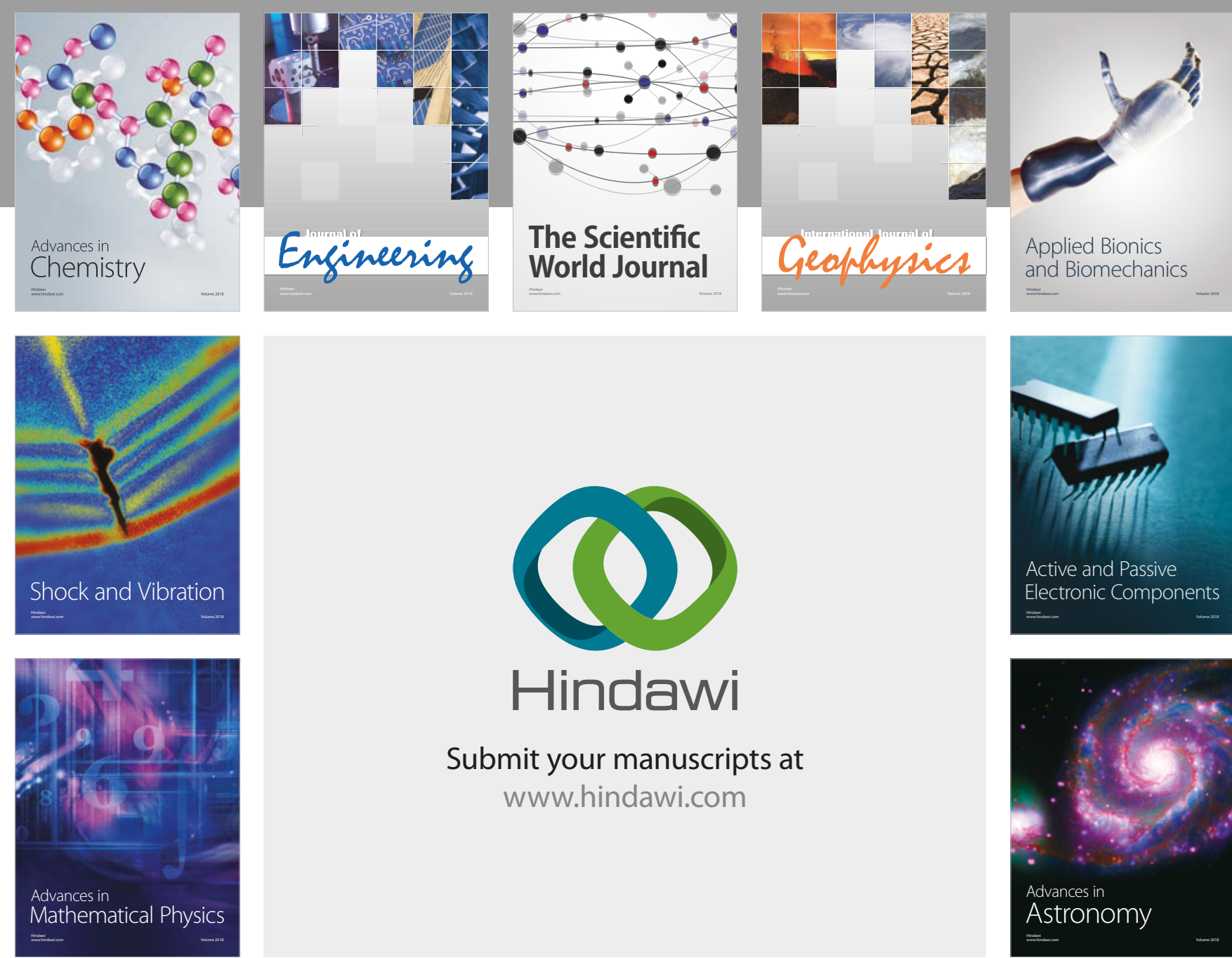

Submit your manuscripts at

www.hindawi.com

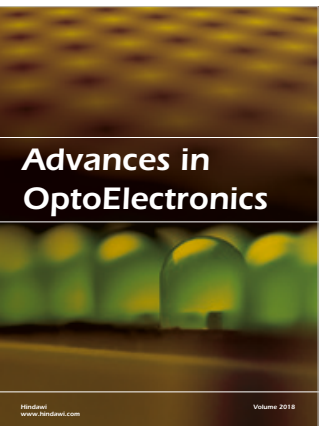

\section{Rotcting Machinery}
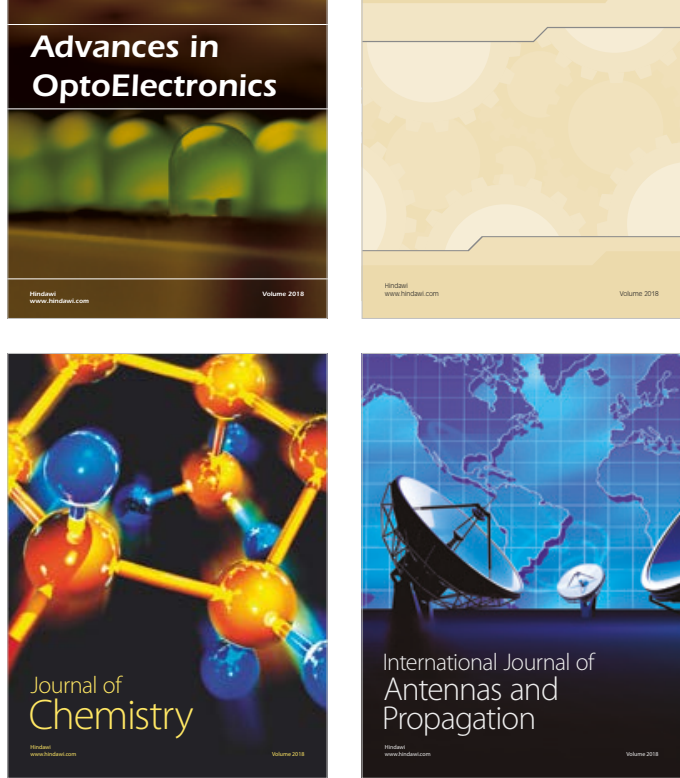

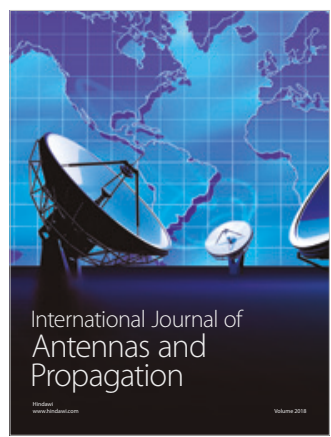

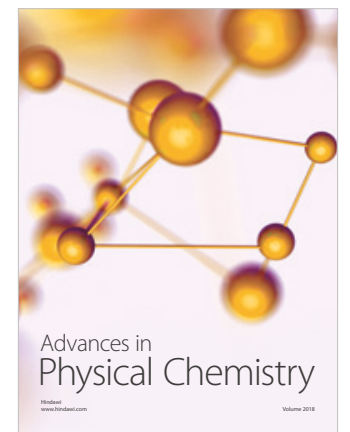

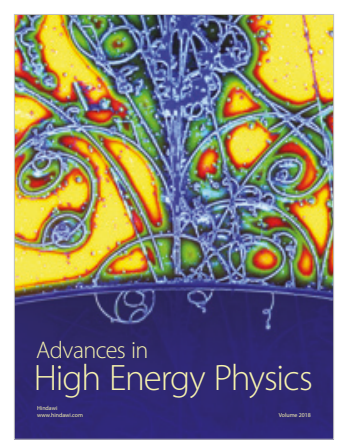

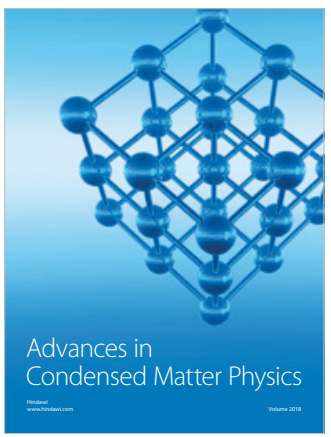

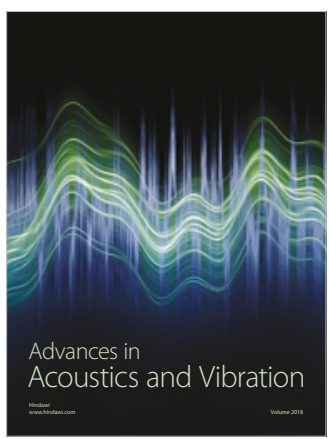

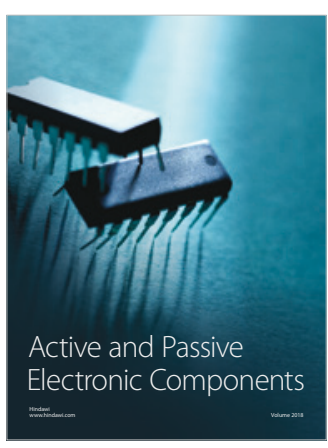
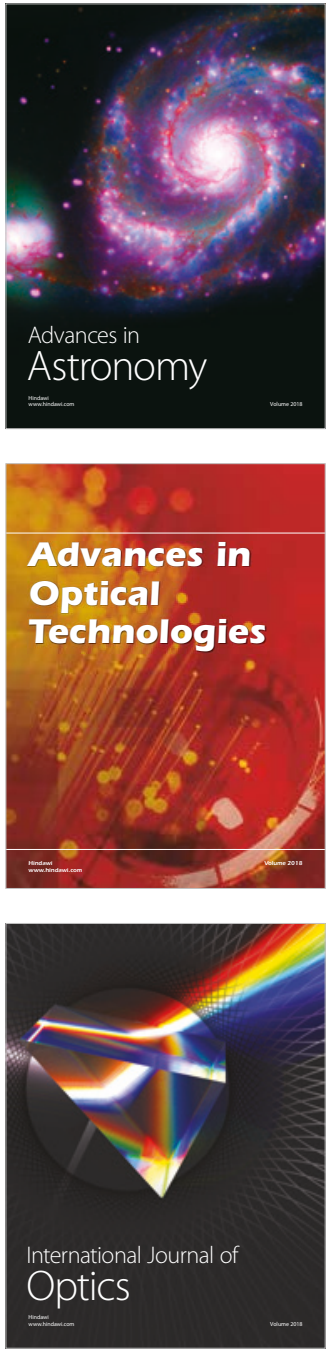